\title{
Review: case management programmes improve patient outcomes
}

Ferguson JA, Weinberger M. Case management programs in primary care.J Gen Intern Med 1998 Feb;13:123-6.

\begin{abstract}
Question
What are the effects of case management programmes on patient centred outcomes, healthcare resource use, and costs? Case management was defined as "a program that uses physician or nonphysician providers to maintain continuous contact with patients via telephone or in-home visits in order to prevent disease exacerbation through intensive assessment and education techniques."
\end{abstract}

\section{Data sources}

English language studies were identified by searching Medline and HealthSTAR (1985-97) using the terms case management, patient care planning, patient-centered care, disease management, care management, and managed care programs. Bibliographies of relevant papers were also reviewed.

\section{Study selection}

Studies were selected if they were randomised controlled trials. Studies were excluded if they did not contain original data; did not include adult patients; or focused solely on discharge planning, inpatient interventions, or treatment of AIDS, malignancy, end stage renal disease, or psychiatric illnesses.

\section{Data extraction}

Data were extracted on the target population, intervention (focus of intervention, general or specialised caregiver, and single or multisite study), sample size, length of follow up, outcome measures, and results.

\section{Main results}

None of the articles identified from the database searches met the inclusion criteria. 9 studies were found through the identification of seminal articles and review of their bibliographies.
Meta-analysis was not done because of the heterogeneity of study designs. 4 studies involved patients with a specific condition (asthma, congestive heart failure, diabetes, or coronary heart disease) and 5 involved patients with a variety of conditions (after discharge from hospital or elderly patients); 3 were done by medical subspecialists and 6 by generalists; and 7 studies were done in single sites and 2 were multisite. Study sample size ranged from 160 to 1400 participants, and length of follow up ranged from 3 months to 1 year.

7 studies that included healthcare utilisation outcomes had conflicting results: 2 found reduced healthcare use with case management programmes, 4 found no differences, and 1 found increased use. No cost savings were found (3 studies). Case management improved clinical outcomes (glycaemic control, smoking cessation, and low density lipoprotein concentrations) (2 studies) and patient satisfaction, quality of life, and functional status (6 studies). None of the interventions that targeted heterogeneous populations or that were done by generalists reduced the use of healthcare resources.

\section{Conclusions}

Case management programmes improve some clinical outcomes, patient satisfaction, quality of life, and functional status, but do not reduce costs. The effect on healthcare utilisation is unclear.

Source of funding: not stated.

For correspondence:DrJA Ferguson, Richard L Roudebush VAMC,HSRED (11H), 1481 West 10th Street, Indianapolis, IN 46202, USA. Fax +1 3175540114.

A modified version of this abstract appears in ACP Journal Club 1998 Sept-Oct.

\section{Commentary}

The goals of case management are improved quality of care and potential cost savings. Research findings on the effect of case management on cost and health outcomes remain inconsistent to date. Furthermore, definitions of case management and its components vary and lack generalisability across settings.

This descriptive synthesis by Ferguson and Weinberger suggests that case management in primary care improves clinical outcomes, but that the effects on costs are yet unknown. The exclusion of trials focused on the primary treatment of other conditions, specifically AIDS and psychiatric illnesses, eliminates a wealth of data that could show positive health and financial outcomes. ${ }^{1}$

5 studies described case management interventions as nurse directed or moni- tored, and 4 studies used interventions involving nurses and doctors or multidisciplinary teams. Nurse directed case management interventions such as patient education, symptom monitoring, telephone follow up, medication review, risk factor reduction, and increased access to health care improved patient satisfaction, quality of life, and functional status. Studies using less rigorous methods also support these findings. ${ }^{2}$

An important question emerges from this study: should case management be instituted on the basis of improved clinical outcomes even if costs are not reduced? Studies citing improved patient, quality, and cost outcomes often focus on specific populations. Until a thorough analysis of the case management literature is available, implementation and evaluation of case management initiatives will necessarily be based on the goals and the discretion of the organisation.

Kylie Nowak RN, BScN Patient Care Process Coordinator, Informatics

Lynn Nagle RN, PhD Managing Director, Informatics Anthony Bernardo RN, BScN Systems Analyst, Informatics Mount Sinai Hospital Toronto, Ontario, Canada

1 Holloway F, Oliver N, Collins E, et al. Case management: a critical review of the outcome literature. European Psychiatry 1995;10:11328.

2 Allred CA, Arford PH, Michel Y, et al. A cost-effectiveness analysis of acute care case management outcomes. Nurs Econ 1995;13: manageme. 